ISBN 978-93-86878-13-7

10th International Conference on Languages, Humanities, Education and Social Sciences (LHESS-18)

Kyoto (Japan) April 19-20, 2018

\title{
Intergenerational Variation in the Perceptions of Korean Monster Dokkaebi
}

\author{
Mikyung Bak \\ Division of Contemporary Culture, Graduate School of Letters, Kyoto University, Japan
}

\begin{abstract}
This article focuses on how Korean people of different age groups typically perceive the visual appearance and personality of Korean mythological monster "dokkaebi" and where such perceptions came from. From September 2015 to September 2017, I conducted a series of surveys to 200 South Korean individuals consisting of four age groups; 50 children aged 10 and under, 50 people in their 10s-20s, 50 people in their 30s40 s, and 50 people aged 50 and over. In the surveys they were asked to answer the following three questions; 1) draw a dokkaebi, 2) describe the dokkaebi's personality, and 3) state from where you derived your perception of the dokkaebi. The answers to the first two questions, described in chapter 1 and chapter 2 respectively, reveal significant intergenerational differences in the perception of the visual appearance and personality of dokkaebi. The answers to the last question, described in chapter 3, indicate that each age group's perceptions were shaped by dokkaebi representations in print media or film content to which they were exposed in their childhood.
\end{abstract}

Keywords: Dokkaebi, Oni, Visual Representation, Media, Piracy

\section{Introduction}

This article focuses on how Korean people of different age groups typically perceive the visual appearance and personality of Korean mythological monster dokkaebi and where such perceptions came from. Before moving on to the main part of the analysis, I first outline the characteristics of dokkaebi and my motive for analyzing this issue.

The Korean word dokkaebi has a very broad meaning that can refer to ghost and monster in general. Dokkaebi can be equated with the kinds of supernatural and mythological entities such as goblin, imp, troll, dwarf and immortal gods. Folktales featuring dokkaebi exist throughout the Korean peninsula [1], and they have become icons of the Korean people and culture; in other words, they are very familiar characters to Koreans.

The visual appearance of dokkaebi, as perceived by many Koreans today, closely resembles the Japanese people's depictions of oni [2]; both creatures are typically depicted with horned heads, spiked clubs, and protruding fangs. They wear fur trousers (or straw skirts) and are naked from the waist up. In my previous work, Kankoku no "oni"-Dokkaebi no shikaku hyōshō [The Visual Representation of Korean Dokkaebi] (November 2015), I highlighted the visual similarities between dokkaebi and oni and analyzed the discourse on this matter that occurred in South Korea during the 1990s [3].

Figure 1, for reference of non-Japanese readers, shows three examples of the visual appearance of oni. The left drawing in a Japanese textbook in 1918 depicts a scene of famous Momotaro folktale where onis are being attacked by Momotaro and his subordinates [4]. The center painting in a Japanese picture book in 1952 depicts a scene of also famous Kobutori folktale where Kobutori, surrounded by onis, is dancing [5]. The right picture, taken by the author in 2014, shows oni character in a Japanese convenience store; you can find this kind of oni characters in many Japanese shops and places in Setsubun season (February 3rd), the end of winter in traditional calendar [6]. 


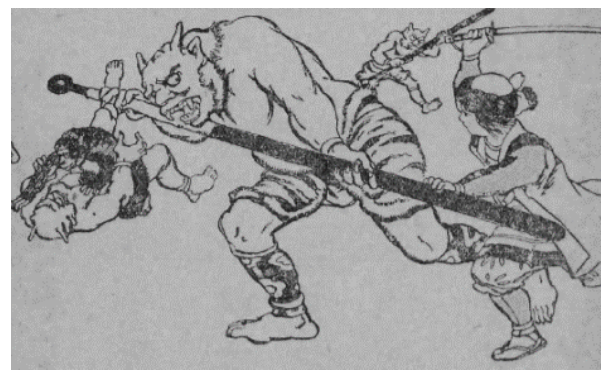

Momotaro in a school text book (1918)

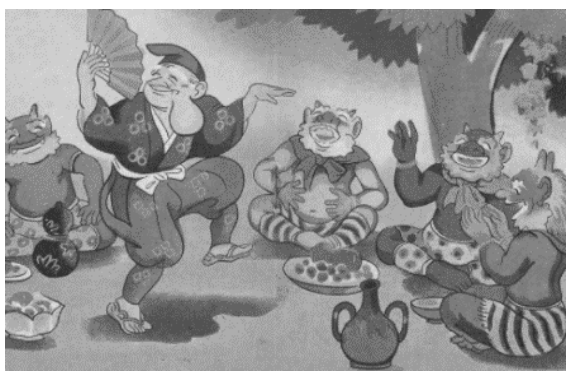

Kobutori in a picture book (1952)

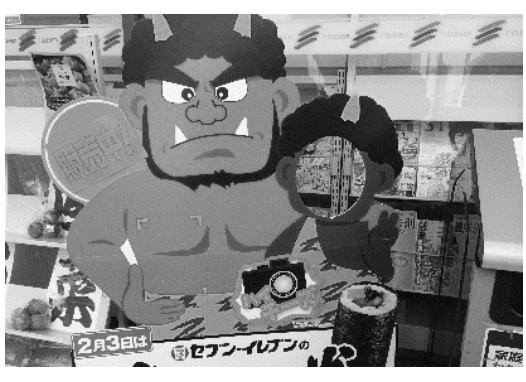

Setsubun event in a convenience store (2014)

Fig. 1: Visual appearance of Japanese oni

In the work mentioned above, I present a September 2015 survey I conducted at a children's art class in South Korea, with the aim of demonstrating how dokkaebi are typically perceived in South Korea today. Specifically, I asked children aged 7-10 to draw a dokkaebi and presented their drawings in the book. These drawings highlight how closely dokkaebi resembles Japanese oni. In addition to presenting the children's drawings of dokkaebi, I also presented the results of two additional questions I asked them: "What does a dokkaebi look like?" and "Where did you see such an image of a dokkaebi?"

Since completing the above work, I have conducted additional surveys among a broader sample of age groups on the same theme. (In each of these surveys, I asked participants to draw a dokkaebi, describe the dokkaebi's personality, and state from where they derived their perception of dokkaebi). Through this work, I obtained data revealing the Korean people's perceptions of dokkaebi (their visual appearance and personalities) and the background circumstances that inspired such perceptions. The data indicates that perceptions of dokkaebi vary by age group, and that most people in each age group derived their perceptions of dokkaebi from picture books and manga they read in their childhood. I therefore posited and tested the hypothesis that intergenerational changes in perceptions of dokkaebi are correlated with changes in media trends. In this article, I summarize this research process.

First, I will describe in more detail the surveys I conducted in South Korea.

A total of 200 individuals participated in the surveys. In addition to 50 children aged 10 and under (in the survey previously mentioned), the study involved 50 people in their $10 \mathrm{~s}-20 \mathrm{~s}, 50$ people in their $30 \mathrm{~s}-40 \mathrm{~s}$, and 50 people aged 50 and over. The ages of the participants indicate their ages at the time of the survey. It should be noted, however, that Korea's traditional system of age reckoning (saeneun nayi) considers an infant to be one year old at birth and adds another year on January 1, thus adding one or two years to the standard system of age reckoning. It should also be noted that there was a period of two years between the first and last survey. (The first survey — among children aged 10 and under — was in September 2015, and the final survey — among people aged 50 and over-was in September 2017).

The question contents were the same in each survey: 1) draw a dokkaebi, 2) describe the dokkaebi's personality, and 3) state from where you derived your perception of dokkaebi. The first two questions inquired into the participants' perceptions of the dokkaebi's visual appearance and personality, while the third question focused on how the participants formed such perceptions (such as by reading picture books or manga). For the first question, I asked participants to draw a picture of what they imagine a dokkaebi to look like, but participants who were not confident in their drawing skills were allowed to describe the dokkaebi in words.

Regarding the survey method, I would generally distribute questionnaires with the three questions to participants and ask them to return the completed questionnaires. However, for those aged 10 and under, I would ask teachers or parents to explain the tasks. Additionally, with participants aged 50 or over (particularly in their 70s-90s), I would in many cases communicate the questions orally and transcribe the answers. 
In the following three chapters, I outline the age group-specific results for each of the three questions and discuss the trends particular to each age group, along with intergenerational variations.

\section{Visual Appearance of Dokkaebi}

Regarding the first question ("draw a dokkaebi"), in Figure 2, I have shown for each age group four dokkaebi drawings that typify the drawings of the age group concerned. (Please note that I have omitted those aged 50 and over because most of the responses from this age group were oral).

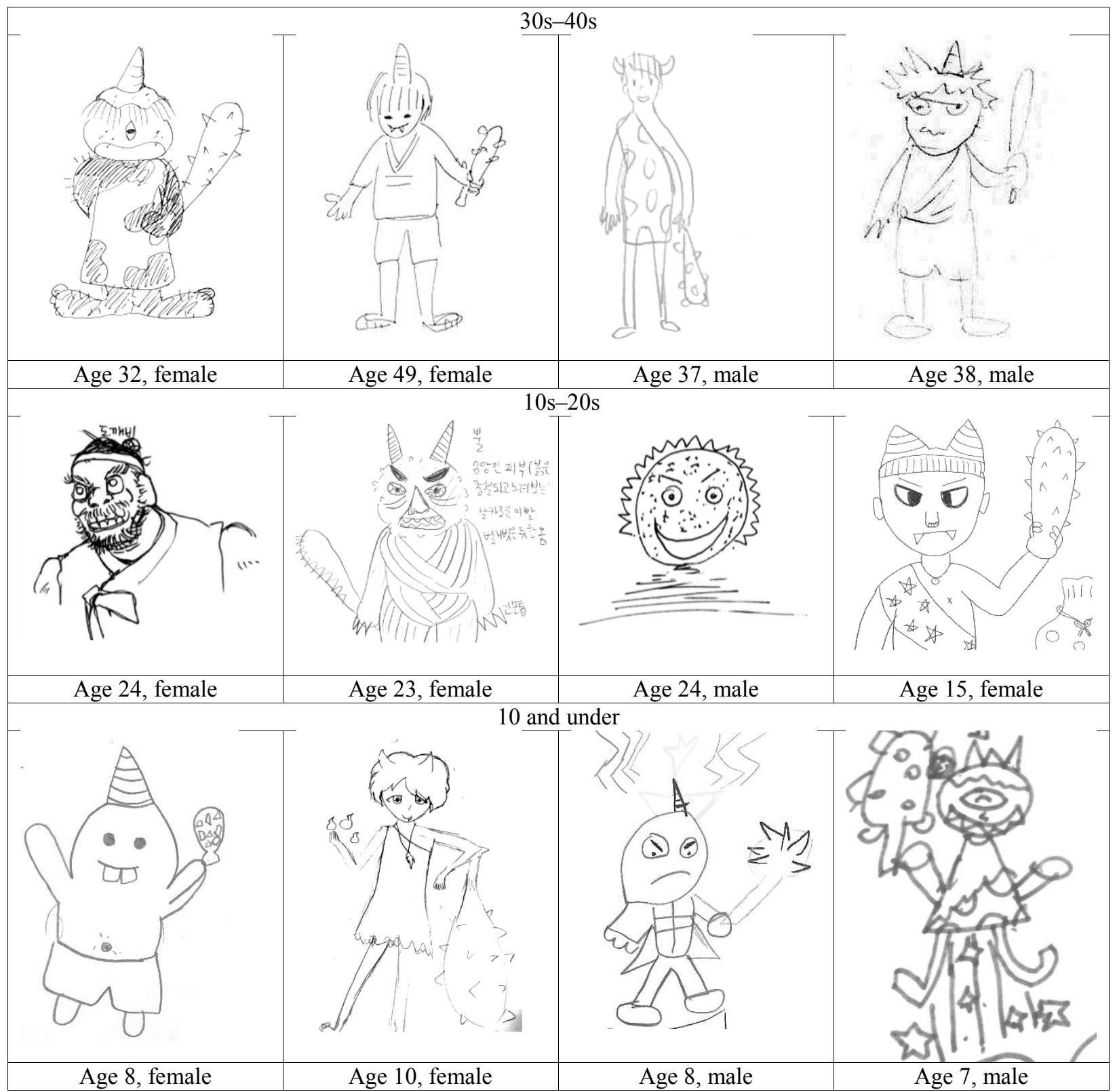

Fig. 2: Visual appearance of a dokkaebi according to each age group. (Sets of four drawings selected from each age group.)

A brief glance at the above pictures reveals many elements in common with the Japanese oni. Table I shows the total number of participants in each age group who included a horned head, spiked club, fangs, and fur trousers in their drawing (or oral description) of a dokkaebi. The data reveal age group-specific trends. 
TABLE I: Age Group-specific Results for Oni-like Elements in Dokkaebi Drawings

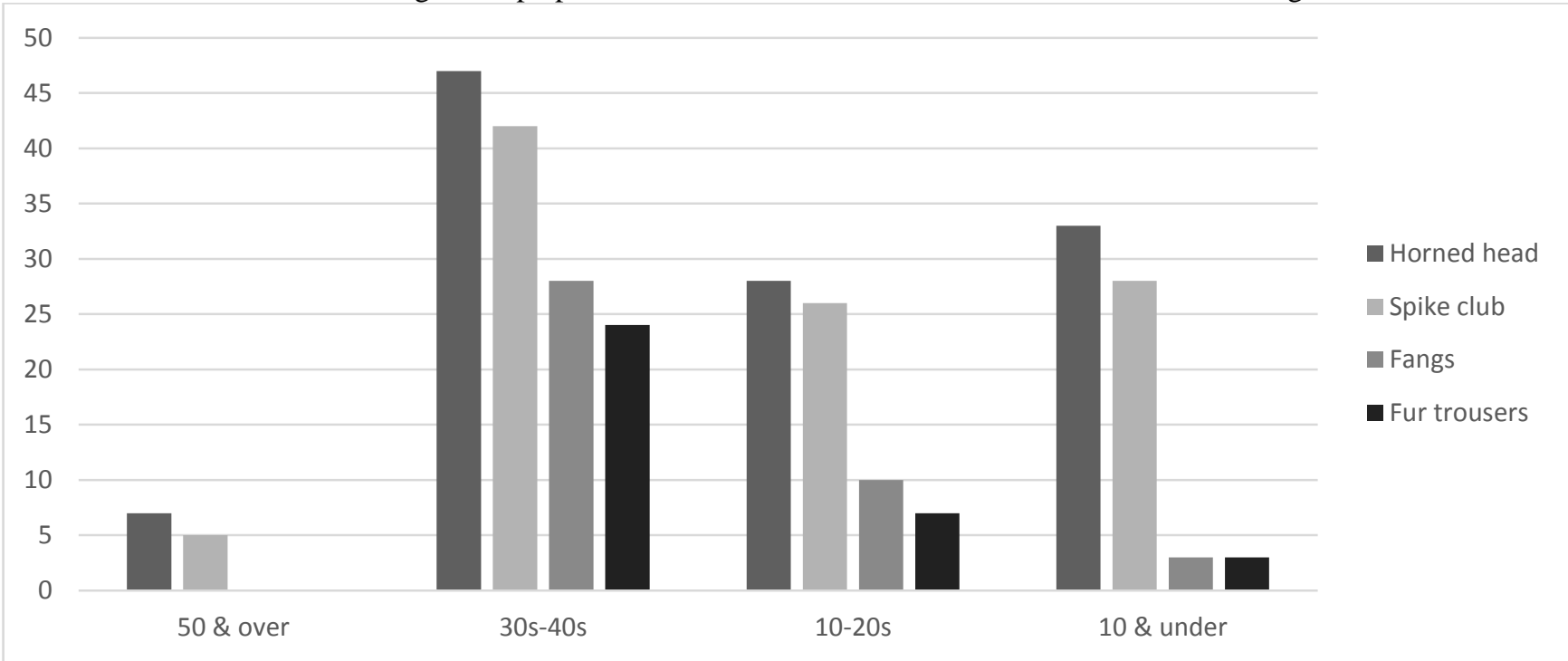

First, many of the participants aged 50 and over (particularly the elderly) did not provide a detailed image; those who did depicted or described an ethereal being with no tangible form, like a ghost light. Seven of the participants in their 50s-60s depicted a dokkaebi with a horned head, spiked club, and other elements common to Japanese oni; these participants reported that they had seen such depictions in manga.

Of all the age groups, the oni features (horned head, spiked club, fangs, fur trousers) were depicted most frequently by the 30s-40s age group, suggesting that people in this group were the most influenced of all the age groups by Japanese depictions of oni. People in this age group would have been children during the period of military rule (1960-1993), during which time South Korea's publishing culture was stagnant. Against this backdrop, the country was awash in pirated versions of Japanese manga, and this content probably shaped these participants' perceptions.

However, if we look at the results for the 10s-20s and 10 and under age groups, we find that although there are still solid numbers of participants whose drawings feature oni-like elements, these numbers are lower than they are in the 30s-40s age group. Fangs and fur trousers, in particular, are much less frequent in these age groups.

One notable characteristic of the 10 and under group's drawings is their diversity. For example, $35 \%$ of these participants depicted the dokkaebi as female, which represents a major intergenerational change, considering that almost all the participants in older age groups depicted the dokkaebi as male. The clothing is more diverse too; in addition to drawings that feature fur trousers or a grass skirt, there are drawings showing dokkaebi in regular human attire. Moreover, in the drawings that feature them, elements like the horned head and fangs are also more diverse. For example, approximately $70 \%$ of the children depicted their dokkaebi with a horned head, yet of these, $50 \%$ drew a single horn, $25 \%$ drew a pair of horns, and $25 \%$ drew three horns. This trend is very interesting given that none of the older participants drew a three-horned head.

\section{Personality of Dokkaebi}

Table II shows the age group-specific results for the second question (“describe the dokkaebi's personality"). Participants cited traits such as scary, cute, funny, stupid, heroic, affectionate, cheerful, and divine/immortal. The trait composition of each age group is represented by the stacked bar charts shown in the table. Where participants cited more than one trait, I included all of them; therefore, the totals for each age group differ. The data reveal intergenerational variation in perceived personality. 
Starting with the 50 and over group, we see that a very large proportion of the participants cited the scary trait. While not counted in the total above, many of the participants in this age group said that since a dokkaebi does not exist in reality, they could not describe its personality. When we consider these participants' responses along with the others, we get the sense that this age group tends to perceive dokkaebi as supernatural beings or fearsome creatures.

TABLE II: Age Group-specific Perceptions of a Dokkaebi’s Personality

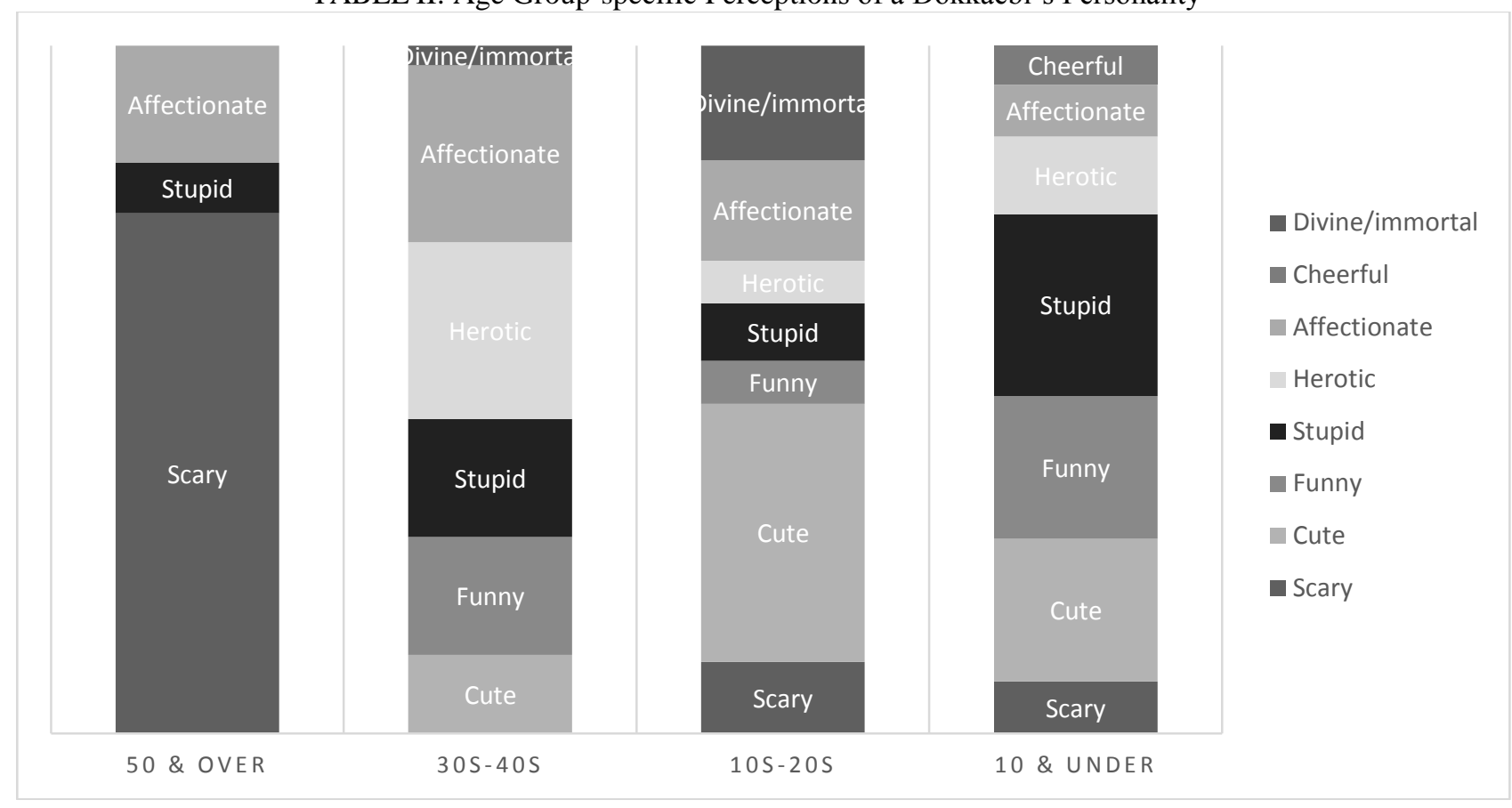

Regarding the 30s-40s age group, almost all of the participants considered the dokkaebi to be a friendly, unintimidating being, citing traits like cute, funny, stupid, heroic, and affectionate. One of the traits cited in this group is divine/immortal, which could be construed as fearsome; however, this trait accounted for only a negligible proportion of the responses, and there were no "scary" responses at all.

Turning to the 10s-20s and 10 and under age groups, as with the visual depictions, a wide range of personality traits was cited. Some of these participants cited scary and divine/immortal, while others cited affectionate, heroic, stupid, funny, and cute. The divine/immortal and cute traits were particularly well represented among the 10s-20s age group, while stupid was a popular trait to cite among the 10 and under age group. At the very least, we can say that there is a much broader array of personality traits associated with dokkaebi.

The divine/immortal trait accounted for a relatively large proportion of responses among the 10s-20s age group. The responses of this age group may have been influenced by a Korean drama series about dokkaebi, which aired from December 2016 to January 2017, only several months before the age group was surveyed (in August-October 2017). Titled Sseulsseulhago Chanlanhasin - Dokkaebi [English title: Guardian: The Lonely and Great God], the TV series features a dokkaebi who appears in human form but whose spirit indwells a sword. Thus, the series depicts the dokkaebi as an immortal being with supernatural powers.

\section{Sources that Inspired the Perceptions}

Table III shows the age group-specific results for the third question ("state from where you derived your perception of dokkaebi"). As with the responses for personality traits, in which participants cited more than one 
source, I included all of the traits; therefore, the totals for each age group differ. A glance at the table reveals that the younger age groups derived their perceptions from a broader array of sources than the older age groups did.

Looking first at the age 50 and over group, manga was a popular response among those in their 50s (5/7 cited this source), and it also featured among the responses of those in their $60 \mathrm{~s}(2 / 11)$. The other responses in this age group were "no source," "oral tradition," and "personal encounter." I had anticipated that many of these participants would cite oral tradition, but I was surprised at how many participants in their 80s and 90s had formed their perception of a dokkaebi based on a personal encounter. In any case, only seven of the participants in this age group cited print media such as manga, and none of them cited video sources such as anime or drama series. This trend is consistent with the fact that only a few of these participants provided drawings of dokkaebi in the previous question.

TABLE III: Age Group-specific Results for Sources that Inspired Dokkaebi Perceptions

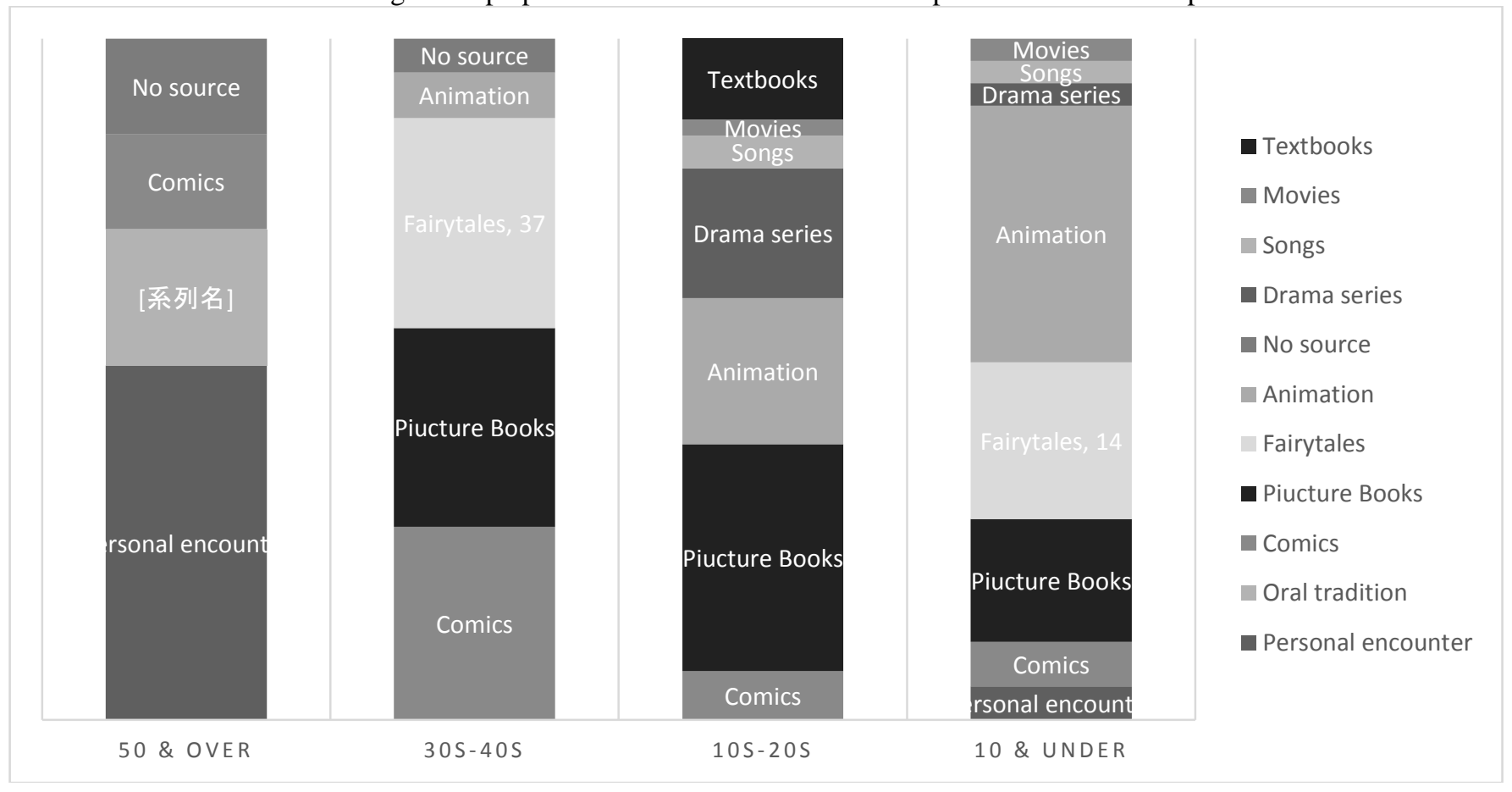

I examined the children's books that were available during the period when the 50s age group were children, and I identified only a few books from this time that featured dokkaebi, as inferred from their titles or other information. I confirmed seven such books in total—one per year from 1964 to 1967, two from 1968, and one from 1969. This finding is consistent with the lack of "children's fairytales" and "picture book" responses. As a follow-up question, I asked participants whether they had seen depictions of dokkaebi in manga or anime. Their answers suggest that their perceptions are inspired more by their own childhood experiences than by print media or film content they viewed later in life. For example, one participant said, "I would see pictures of dokkaebi when I was parenting and when I was looking after my grandchildren, but these pictures were made for children; they were not like real dokkaebi."

Conversely, in the 30s-40s age group, the responses comprise "anime," "children's fairytales," "picture books," and "manga," which suggests that perceptions of dokkaebi changed significantly from the previous generation. On the other hand, the 30s-40s age group, compared to younger age groups, featured a greater proportion of participants who cited print media such as picture books and manga.

As I mentioned in Chapter 2, this age group was the most influenced, among all the age groups, by Japanese oni depictions. This trend is consistent with the fact that when these participants were children, the dokkaebi 
they saw in popular picture books, fairytales, and anime circulating at the time in South Korea were all oni images. The influence of manga is also noticeable among responses from participants in their 50s, but this influence was most keenly felt by those in their 30s and 40s, and the perceptions of these participants were strongly shaped by such material. This age group was more likely than the previous generation to perceive dokkaebi as friendly, non-intimidating beings (as illustrated by responses like "cute," "funny," and "stupid"), and such perceptions likely stem from the oni/dokkaebi depicted in manga as cartoonish characters.

As we move down to the $10 \mathrm{~s}-20$ s and 10 and under age groups, we notice an increasingly large proportion of participants citing film content such as movies, drama series, and anime. That said, the above-mentioned TV series, "Guardian: The Lonely and Great God," may have had the most impact on the 10s-20s age group. Interestingly, the responses of the 10s-20s age group included "textbooks," and those of the 10 and under age group included "personal encounter." However, when the children cited "personal encounter," they meant encountering an actor impersonating a dokkaebi at a cultural or amusement facility (such as the dokkaebi Museum in Jangsu, Hwaam Cave in Jeongseon, or Lotte World); this is quite different from the elderly participants" "personal encounters," which denoted supernatural experiences.

In particular, the dokkaebi characters drawn by children 10 and under retained to some extent the oni-like elements (such as a horned head and spiked club) and possessed a diverse array of features, including different genders, clothing, and number of horns. Such diversity is consistent with the fact that dokkaebi content designed for children today features many more innovative representations of dokkaebi. It is also consistent with the fact that today's children have more varied opportunities to encounter dokkaebi in the first place; in addition to seeing dokkaebi characters in print material, they can also encounter them in amusement facilities, for example.

\section{Conclusion}

In this article, I reported survey results showing how different age groups perceive the visual image and personalities of dokkaebi-Korea's version of mythological monster, which have become a cherished icon of Korean culture - and discussed the trends particular to each age group, as well as intergenerational variations. I then examined the factors that gave rise to such intergenerational variation; specifically, I examined how each age group's perceptions were shaped by dokkaebi representations in print media or film content to which they were exposed in their childhood.

Due to space constraints, I am unable to present the comprehensive results of my surveys, having had to abridge my discussion of transitions over time in print media and film. I intend to present the results and analysis in more detailed way at a later date. For the time being, however, I believe this article provides a general overview of how today's Korean people perceive dokkaebi and how print media and film content have influenced such perceptions.

\section{Reference}

[1] There is some regional variation in pronunciation; varieties include dochabi (Jeolla province), dokabi (Gyeongsang province), and dochebi (Jeju Island, Namhae).

[2] Michio Nmekawa, Momotarozō no Henyō [The Process of Change in the Image of Momotaro], Japan: Tokyo Shoseki, 1981, p. 41.

[3] Mikyung Bak, Kankoku no "oni"-Dokkaebi no shikaku hyōshō [The Visual Representation of Korean Dokkaebi], Japan: Kyoto University Press, 2015.

[4] Monbushō [Ministry of Education], Jinjo Shogaku Kokugo Dokuhon [Japanese Reader in Elementary School], Japan: Monbushō, 1918, p.52.

[5] Hakuyo Furuya, Hanashi-no ehon [Picture Book of Stories],1952, Japan: Masumi Shobo, pp. 5-6.

[6] Photo taken by Mikyung Bak, February 2014. 